\title{
Numerical simulation of flow past a heated/cooled sphere
}

\author{
$\operatorname{AUTHOR}(\mathrm{S})$ :
}

Kurose, Ryoichi; Anami, Mamiko; Fujita, Akitoshi; Komori, Satoru

\section{CITATION:}

Kurose, Ryoichi ...[et al]. Numerical simulation of flow past a

heated/cooled sphere. Journal of Fluid Mechanics 2012, 692: 332-346

\section{ISSUE DATE:}

2012-02

URL:

http://hdl.handle.net/2433/154559

\section{RIGHT:}

C Cambridge University Press 2012; この論文は出版社版でありません 。引用の際には出版社版をご確認ご利用ください。; This is not the published version. Please cite only the published version. 


\title{
Direct numerical simulation of flow past a heated/cooled sphere
}

\author{
RYOICHIKUROSE†, MAMIKOANAMI, \\ AKITOSHI FUJITA AND SATORUKOMORI \\ Department of Mechanical Engineering and Science, and Advanced Research Institute of Fluid \\ Science and Engineering, Kyoto University,Yoshida-honmachi, Sakyo-ku, Kyoto 606-8501, \\ Japan
}

(Received ?? and in revised form ??)

The characteristics of flow past a heated/cooled sphere are investigated for $50 \leqslant R e_{p} \leqslant$ 400 in conditions with and without buoyancy by means of three-dimensional direct numerical simulation (DNS) in which temperature dependencies of fluid properties such as density and viscosity are exactly taken into account. The results show that in the absence of buoyancy, drag coefficients of the heated and cooled spheres are larger and smaller than those of the adiabatic case, respectively, and Nusselt numbers of them are smaller and larger than the values estimated by a widely used empirical expression for predicting Nusselt numbers, respectively. In addition, the temperature difference between the sphere and ambient fluid strongly affects the flow separation points, size of vortex ring behind the sphere and Strouhal number for vortex shedding. These changes are attributed to the temperature dependencies of fluid properties in the vicinity of the sphere. Even in the presence of buoyancy, temperature dependencies of fluid properties strongly affect drag coefficient and Nusselt number and therefore Boussinesq approximation becomes inapplicable as the temperature difference increases, regardless of the magnitude of Richardson number.

Key Words: flow past a heated/cooled sphere, drag coefficient, Nusselt number, buoyancy

\section{Introduction}

Heat transfer between dispersed particles/droplets and fluid is often seen in industrial flows such as spray and pulverized coal combustion (e.g. Nakamura et al 2005; Baba \& Kurose 2008; Kurose et al. 2009). It is therefore of great importance to understand the characteristics of flow past a heated/cooled sphere in effectively designing and operating such industrial equipments.

Kurose \& Komori (1999) and Kurose et al. (2003) applied direct numerical simulation (DNS) to flow past a stationary sphere and investigated drag, lift and scalar transfer from the sphere surface from the viewpoint of droplet evaporation. Bagchi \& Kottam (2008) performed similar DNS and studied the effect of freestream turbulence on the heat transfer from the sphere surface to ambient fluid. Also, Kurose et al. (2009) applied the DNS to flows both inside and outside a sphere and investigated the relation between the heat transfer and droplet evaporation in detail. However, it should be noted that in these studies heat was treated as a passive scalar which does not affect the flow structure.

$\dagger$ Email address for correspondence: kurose@mech.kyoto-u.ac.jp 
On the other hand, Mansoorzadeh et al. (1998) and Kotouč et al. (2009) examined the characteristics of flow past a heated/cooled sphere in the presence of buoyancy due to the gravity and showed that buoyancy causes the changes in drag and separation points. However, these studies are insufficient to exactly evaluate the effect of temperature difference between the sphere and ambient fluid because the temperature dependencies of fluid properties such as density and viscosity were neglected and the Boussinesq approximation which assumes small temperature difference was employed.

The purpose of this study is therefore to investigate the characteristics of flow past a heated/cooled sphere in both conditions with and without buoyancy due to the gravity by performing DNS in which the temperature dependencies of fluid properties are exactly taken into account.

\section{Direct numerical simulation (DNS)}

\subsection{Flow configuration and governing equations}

The flow geometry and coordinate system for the computations are shown in figure 1. A stationary rigid sphere with diameter $d$ is placed at the origin $(0,0,0)$ of the center of a spherical domain with diameter of $45 d$. The imposed flow is a uniform air flow past the sphere. The governing equations are three-dimensional continuity, Navier-Stokes (NS), energy conservation equations and equation of state written as

$$
\begin{gathered}
\frac{\partial \rho}{\partial t}+\nabla \cdot(\rho \boldsymbol{u})=0 \\
\frac{\partial}{\partial t} \rho \boldsymbol{u}+[\nabla \cdot \rho \boldsymbol{u} \boldsymbol{u}]=-\nabla p-[\nabla \cdot \boldsymbol{\tau}]+\rho \boldsymbol{g}, \\
\frac{\partial}{\partial t} \rho h+(\nabla \cdot \rho h \boldsymbol{u})=-(\nabla \cdot \lambda \nabla T), \\
p=\rho R T,
\end{gathered}
$$

where enthalpy $h$ and stress tensor $\tau$ are given by

$$
\begin{gathered}
h=\int C_{p} d T \\
\boldsymbol{\tau}=-\left(p+\frac{2}{3} \mu \nabla \cdot \boldsymbol{u}\right) \boldsymbol{I}+\mu\left[\nabla \boldsymbol{u}+(\nabla \boldsymbol{u})^{\mathrm{T}}\right] .
\end{gathered}
$$

Here, $\boldsymbol{u}(=(u, v, w))$ is the fluid velocity vector, $p$ the pressure, $\boldsymbol{g}$ the gravity, $T$ the temperature, $R$ the universal gas constant, $\rho$ the density, $\mu$ the viscosity, $\lambda$ the thermal conductivity, $C_{p}$ the specific heat, I the unit tensor, and the superscription T the transposed matrix. In this study, the values of $\rho, \mu, \lambda$ and $C_{p}$ were varied with temperature. The temperature dependencies of $\rho, \mu$ and $C_{p}$ were computed by equation (2.4), Sutherland's formula and NASA polynomials, respectively, whereas that of $\lambda$ was given under the assumption that Prandtl number $\operatorname{Pr}\left(=C_{p} \mu / \lambda\right)$ keeps a constant value $(=0.72)$.

These equations were solved directly using the FrontFlow/Red (FFR) code which is based on a finite volume method for unstructured grids. The convective terms of the NS equation and the energy conservation equation were approximated by the secondorder accurate central difference method and the third-order accurate upwind difference method, respectively. The third-order accurate Adams-Moulton method and the Euler method were implicitly used to advance the NS equation and the energy conservation equation in time, respectively. 


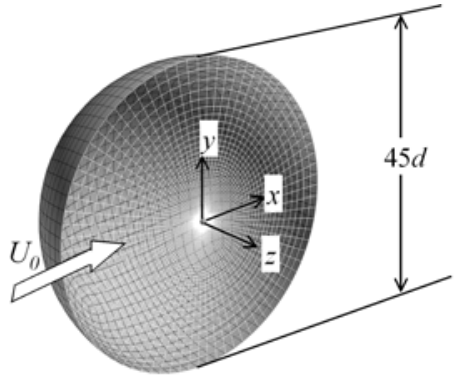

Whole region

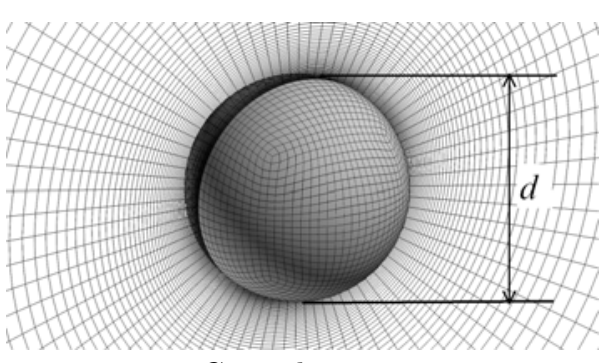

Central region

Figure 1. Computational domain and grids.

\subsection{Boundary conditions}

The inlet boundary conditions for $x<0$ are given by the Dirichlet condition as

$$
\begin{gathered}
u=U_{0}, v=w=0, \\
T=T_{0},
\end{gathered}
$$

and the outlet boundary conditions for $x>0$ are given by the Neumann condition as

$$
\begin{aligned}
& \frac{\partial \boldsymbol{u}}{\partial x}=0, \\
& \frac{\partial T}{\partial x}=0 .
\end{aligned}
$$

The boundary conditions on the surface of the sphere are given by the non-slip and constant-temperature conditions as

$$
\begin{gathered}
u=v=w=0, \\
T=T_{\text {sphere }} .
\end{gathered}
$$

\subsection{Numerical conditions}

The DNS was applied to flow past a heated/cooled sphere in both conditions with and without buoyancy due to the graviy. In addition, only in the condition with buoyancy, the validity of the Boussinesq approximation which is often used to simplify the governing equations under the assumption of constant fluid properties was examined.

In the absence of buoyancy, the calculations with the variable fluid properties were performed for $R e_{p}=50,100,200,400$ and the temperature conditions of the heated sphere of $T_{\text {sphere }}=1000 \mathrm{~K}$ and $T_{0}=293 \mathrm{~K}$, the cooled sphere of $T_{\text {sphere }}=293 \mathrm{~K}$ and $T_{0}$ $=1000 \mathrm{~K}$ and the adiabatic case of $T_{\text {sphere }}=T_{0}=293 \mathrm{~K}$, provided that $d=1 \mathrm{~mm}$.

In the presence of buoyancy, on the other hand, the gravity $\boldsymbol{g}\left(=\left(-9.8 \mathrm{~m} / \mathrm{s}^{2}, 0,0\right)\right)$ was exerted in the opposite direction of the main stream. By introducing the relation of $-\nabla p=-\nabla p_{0}-\rho_{0} \boldsymbol{g}$, equation (2.2) becomes

$$
\rho \frac{D \boldsymbol{u}}{D t}=-\nabla p_{0}-[\nabla \cdot \boldsymbol{\tau}]+\left(\rho-\rho_{0}\right) \boldsymbol{g} .
$$

Here, the application of the Boussinesq approximation to equation (2.13) leads to

$$
\rho_{0} \frac{D \boldsymbol{u}}{D t}=-\nabla p_{0}-[\nabla \cdot \boldsymbol{\tau}]-\rho_{0} \boldsymbol{g} \beta\left(T-T_{0}\right),
$$

where $\rho_{0}$ is the characteristic density, namely the density of the inlet flow and $\beta\left(=1 / T_{0}\right)$ 
is the coefficient of thermal expansion. For the calculations with the Boussinesq approximation, equation (2.14) was solved instead of equation (2.2). For both cases with the variable fluid properties and the Boussinesq approximation, particle Reynolds number $R e_{p}\left(=\rho_{0} U_{0} d / \mu_{0}\right)$ was 100 , and Richardson number $R i\left(=\beta|\boldsymbol{g}|\left(T_{\text {sphere }}-T_{0}\right) d / U_{0}^{2}\right)$ ranged from -0.1 to 0.5 . Here, the positive and negative $R i$ represent the heated and cooled sphere, respectively. It should be noted here that only for the calculations with the variable fluid properties, $d$ and $\Delta T\left(=T_{\text {sphere }}-T_{0}\right)$ need to be determined because these parameters change the fluid properties even for the same $R i$. Hence, $T_{0}$ and $T_{\text {sphere }}$ of the heated sphere were set to be $293 \mathrm{~K}$ and 293-985 K, respectively, and those of the cooled sphere were set to be $990 \mathrm{~K}$ and $285-990 \mathrm{~K}$, respectively, provided that $d=1,5,10$, $50 \mathrm{~mm}$.

\section{Results and discussion}

\subsection{Absence of buoyancy}

\subsubsection{Vortex behavior}

Figures 2 and 3 show the distributions of instantaneous streamlines and temperature on the $(x, y)$-plane for $R e_{p}=50$ and 200, respectively. The behavior of the vortex ring behind the sphere is observed to be strongly affected by the temperature difference between the sphere and ambient fluid. In order to quantitatively evaluate the vortex ring, the position of the vortex centers $l^{*}$, distance between the vortex centers $c^{*}$ and size of the vortex ring $s^{*}$ against particle Reynolds number $R e_{p}$ are shown in figure 4, together with the experimental results by Taneda (1956). All lengths are non-dimensionalized by $d$. It is found that for the adiabatic case the predicted characteristics of the vortex ring are in good agreement with those by the experiments (1956). At $R e_{p}=200, l^{*}, c^{*}$ and $s^{*}$ of the heated sphere are all larger than those of the adiabatic case, whereas those of the cooled sphere are all smaller. At $R e_{p}=50$, on the other hand, $l^{*}, c^{*}$ and $s^{*}$ of both the heated and cooled spheres are smaller than those of the adiabatic case. These changes in the size of the vortex ring are considered to be caused by the changes in velocity boundary layer thickness and separation point, as described below.

Figure 5 shows the velocity boundary layer thickness $\delta^{*}$ against particle Reynolds number $R e_{p}$. Here, $\delta^{*}$ is given by non-dimensionalizing the average of the distance from the sphere surface to the location indicating $u=0.99 U_{0}$ in the range of $-0.05 d<$ $x<0.05 d$. Compared to the adiabatic case, $\delta^{*}$ of the heated and cooled spheres are thicker and thinner, respectively, because the viscosity around the heated and cooled spheres are higher and lower than that of the adiabatic case, respectively. The change in $\delta^{*}$ substantially acts to change the effective diameter of the sphere, consequently the effective particle Reynolds number. Therefore the thicker velocity boundary layer (i.e. the increase in the effective particle Reynolds number) of the heated sphere increases the size of vortex ring, whereas the thinner velocity boundary layer (i.e. the decrease in the effective particle Reynolds number) of the cooled sphere decreases it. In terms of the boundary layer flow in the vicinity of the sphere surface, however, the change in the separation point affects the size of vortex in the completely opposite way. Figure 6 shows the separation point $\alpha$ against particle Reynolds number $R e_{p}$. Compared to the adiabatic case, $\alpha$ of the heated and cooled spheres are found to shift to the downstream and upstream, respectively, mainly because of the change in viscosity. This shift of $\alpha$ to the downstream for the heated sphere acts to make the size of vortex ring smaller, whereas the shift of $\alpha$ to the upstream for the cooled sphere acts to make it bigger. Thus, the changes in $\delta^{*}$ and $\alpha$ due to the heat transfer complicate the formation of the vortex 


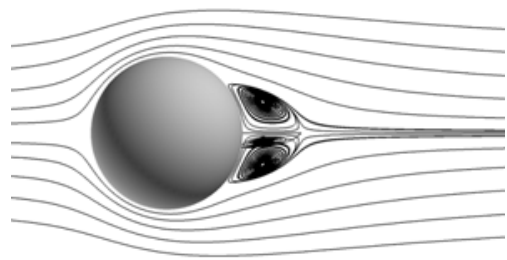

(a) Adiabatic case

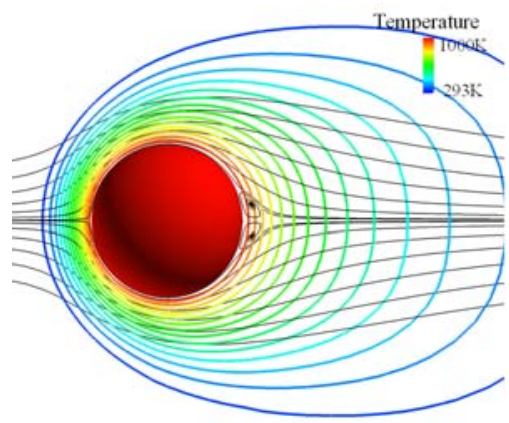

(b) Heated sphere

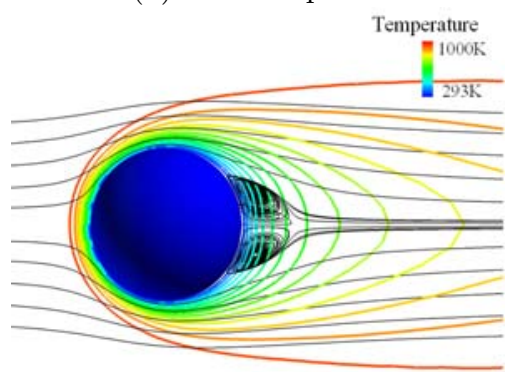

(c) Cooled sphere

FiguRE 2. Distributions of instantaneous streamlines and temperature for $R e_{p}=50$.

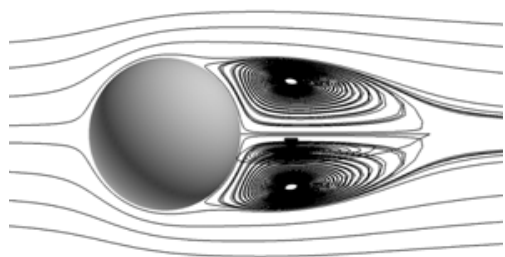

(a) Adiabatic case

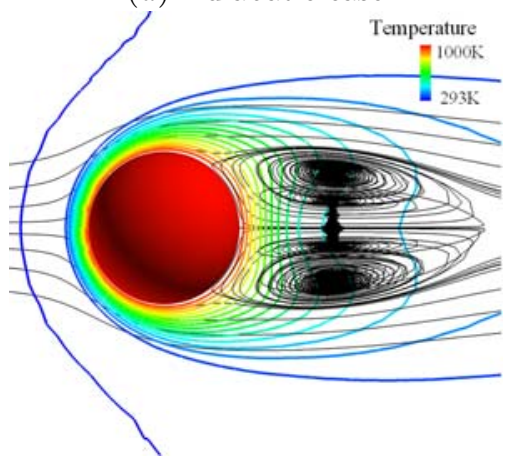

(b) Heated sphere

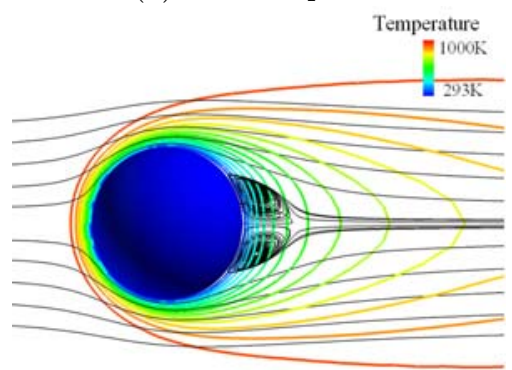

(c) Cooled sphere

FiguRE 3. Distributions of instantaneous streamlines and temperature for $R e_{p}=200$.

ring. It is considered that the reason of the discrepancy in the effects of the temperature difference on the characteristics of the vortex ring between at $R e_{p}=200$ and 50, as described above, is due to the fact that although the characteristics of the vortex ring are affected by the change in $\delta^{*}$ in general, the effect of the change in $\alpha$ becomes large in creeping flows as seen at the extremely low $R e_{p}$.

For $R e_{p}=400$, the Strouhal number $S t$ of vortex shedding was examined. Here $S t$ is given by

$$
S t=\frac{f d}{U_{0}},
$$

where $f$ is the vortex-shedding frequency. $S t$ of the adiabatic case, heated sphere and cooled sphere were $0.13,0.11$ and 0.14 , respectively. This meant that the higher viscosity in the vicinity of the heated sphere prevents vortices from separating from the sphere, whereas the lower viscosity in the vicinity of the cooled sphere has the opposite effect. 


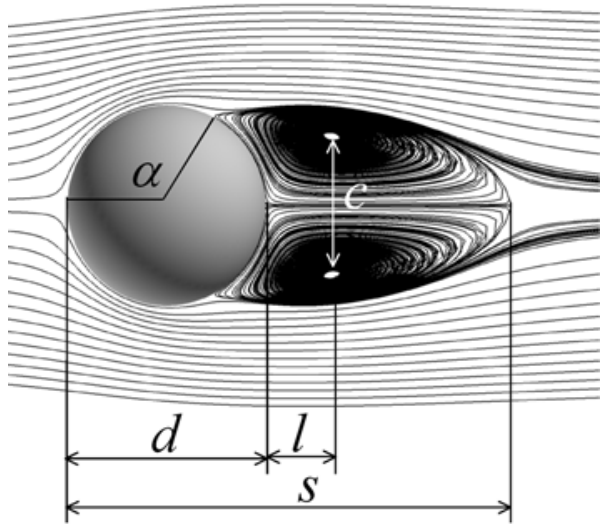

(a) Difinitions of properties

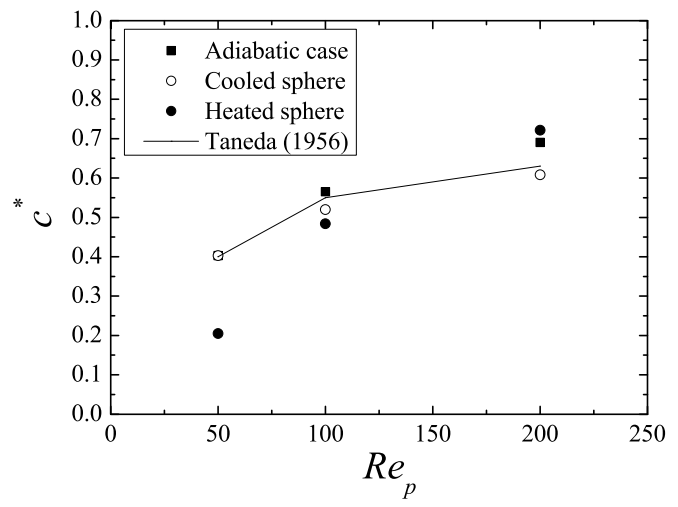

(c) Distance between vortex centers $c^{*}$

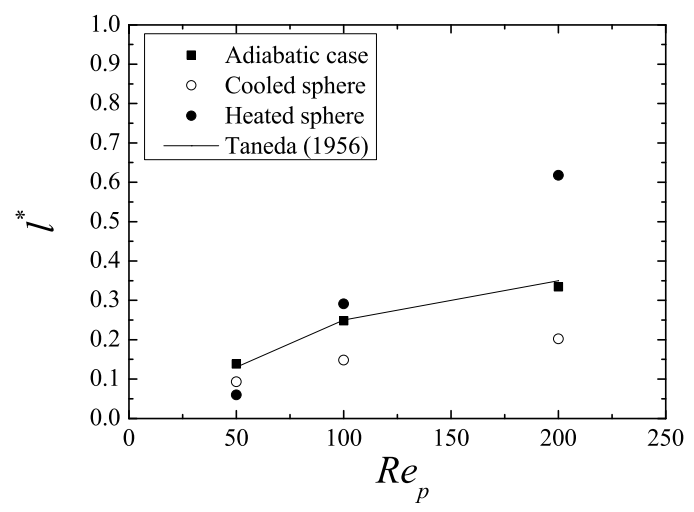

(b) Position of vortex centers $l^{*}$

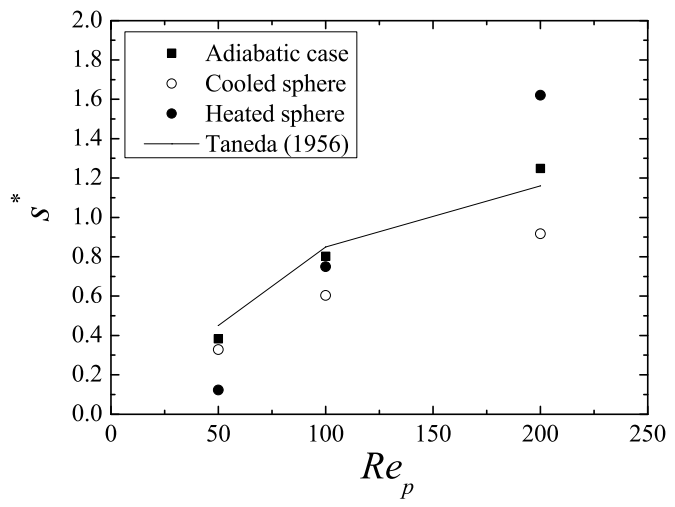

(d) Size of vortex ring $s^{*}$

Figure 4. Characteristics of vortex ring agaist particle Reynplds number $R e_{p}$.

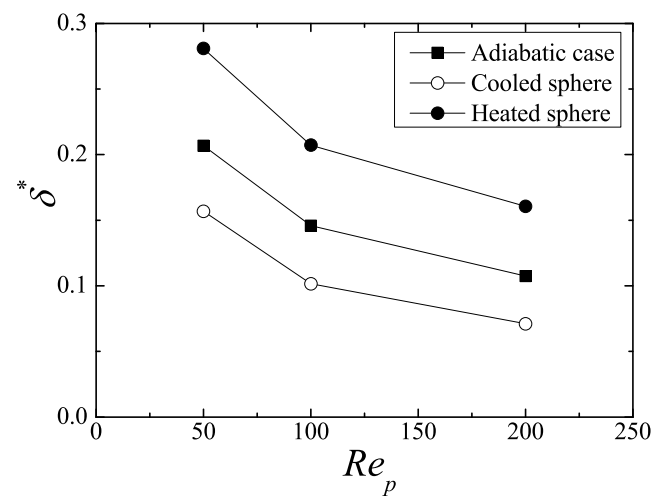

FiguRE 5. Velocity boundary layer thickness $\delta^{*}$ against particle Reynolds number $R e_{p}$. 


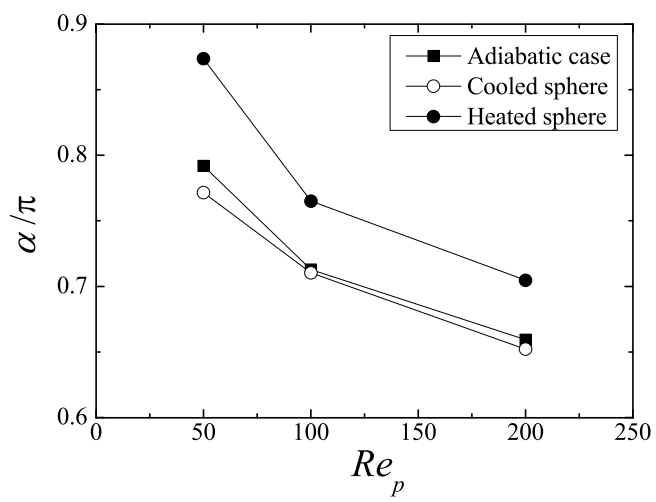

FIGURE 6. Separation point $\alpha$ against particle Reynolds number $R e_{p}$.

\subsubsection{Drag coefficient}

Figure 7 shows the drag coefficients $C_{D}$ of the heated and cooled spheres against particle Reynolds number $R e_{p}$ together with those of the adiabatic case obtaind by the present calculation and those obtained by the empirical correlation of Schiller \& Nauman (1933). Here $C_{D}$ is given by non-dimensionalizing the integrated pressure and viscous stress over the surface of the sphere:

$$
C_{D}=\frac{1}{\frac{1}{2} \rho_{0} U_{0}^{2} A}\left(-\int_{S} p \boldsymbol{e}_{\boldsymbol{x}} \cdot \boldsymbol{n} d S+\int_{S} \boldsymbol{n} \cdot \boldsymbol{\tau} \cdot \boldsymbol{e}_{\boldsymbol{x}} d S\right),
$$

where $A$ is the profile area of the sphere, $\boldsymbol{e}_{\boldsymbol{x}}$ the unit vector in the $x$ direction, and $\boldsymbol{n}$ the unit normal vector to the surface of the sphere. Good agreement is found in $C_{D}$ of the adiabatic case between by the present calculation and by the empirical correlation (1933). It is also found that $C_{D}$ of the heated and cooled spheres are larger and smaller than those of the adiabatic case, respectively. In order to examine the reason of the change in $C_{D}$, the contributions of the friction and pressure, $C_{D, f}$ and $C_{D, p}$, to the drag coefficient $C_{D}$ are shown against particle Reynolds number $R e_{p}$ in figure 8 . In addition, the local friction stress $C_{f}$ and pressure $P^{*}$ on the sphere surface for $R e_{p}=200$ are shown in figure 9 . Here $C_{D, f}, C_{D, p}, C_{f}$ and $P^{*}$ are given by

$$
\begin{gathered}
C_{D, f}=\frac{1}{\frac{1}{2} \rho_{0} U_{0}^{2} A} \int_{S} \boldsymbol{n} \cdot \boldsymbol{\tau} \cdot \boldsymbol{e}_{\boldsymbol{x}} d S, \\
C_{D, p}=\frac{1}{\frac{1}{2} \rho_{0} U_{0}^{2} A}\left(-\int_{S} p \boldsymbol{e}_{\boldsymbol{x}} \cdot \boldsymbol{n}\right) d S, \\
C_{f}=\frac{\boldsymbol{n} \cdot \boldsymbol{\tau} \cdot \boldsymbol{e}_{\boldsymbol{x}}}{\frac{1}{2} \rho_{0} U_{0}^{2}}, \\
P^{*}=\frac{p-p_{\infty}}{\frac{1}{2} \rho_{0} U_{0}^{2}},
\end{gathered}
$$

where $p_{\infty}$ is the pressure in the freestream and $\theta$ in figure 9 is the angle from the fore edge point of the sphere surface on the $(x, y)$-plane. It is found that both the friction and pressure contribute to the change in $C_{D}$. In particular, $C_{f}$ of the heated and cooled spheres are remarkably higher and lower than those of the adiabatic case, respectively, in the region of $\theta<0.7 \pi$ of the sphere surface because the heated fluid has the higher 


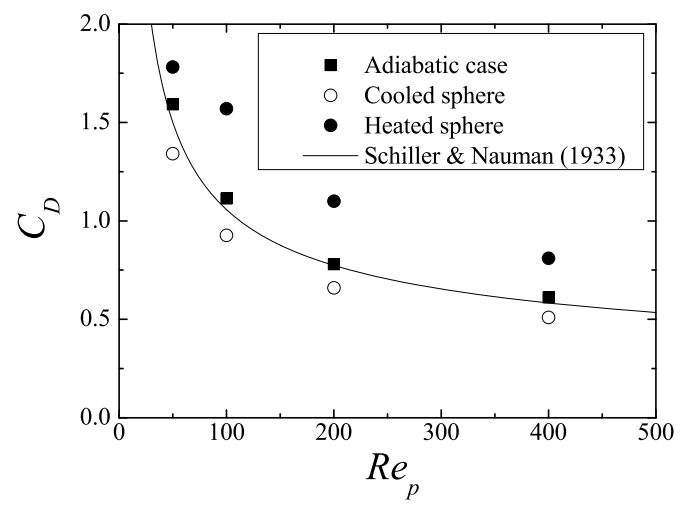

FiguRE 7. Drag coefficients $C_{D}$ against particle Reynolds number $R e_{p}$.

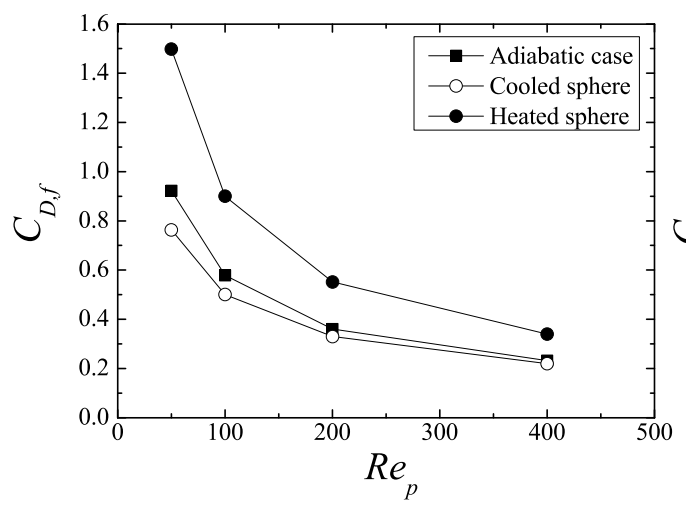

(a) Contribution of friction $C_{D, f}$

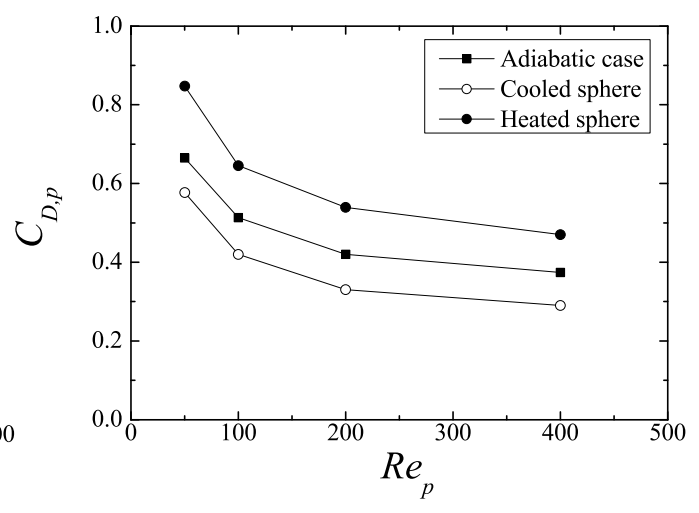

(b) Contribution of pressure $C_{D, p}$

Figure 8. Contributions of the friction and pressure, $C_{D, f}$ and $C_{D, p}$, to the drag coefficient $C_{D}$ against particle Reynolds number $R e_{p}$.

viscosity, whereas the cooled fluid has the lower viscosity, compared to the adiabatic case. On the other hand, the effect of $P^{*}$ on $C_{D, p}$ is eminent in the region of $\theta>0.6 \pi$ on the sphere surface (it should be noted that the reverse effect in the region of $\theta=0.3-0.6 \pi$ is small enough when $x$-component of $P^{*}$ is considered). This change in $P^{*}$ can be explained by the changes in $C_{f}$ and separation point $\alpha$ (see figure 6). Compared to the adiabatic case, $\boldsymbol{\alpha}$ of the heated and cooled spheres shift to the downstream and upstream, respectively. Therefore not only the increase in $C_{f}$ but also the longer distance to the separation point for the heated sphere results in the consumption of more energy and hence the decrease in the pressure behind the sphere, and the exactly opposite phenomenon occurs for the cooled sphere.

\subsubsection{Nusselt number}

Figure 10 shows the Nusselt numbers $N u$ against particle Reynolds number $R e_{p}$ together with those obtained by the widely used empirical correlation of Beard \& Pruppacher (1971) where small temperature difference of several tens of Kelvin is given. Here $N u$ is given by non-dimensionalizing the integrated temperature gradient over the surface 


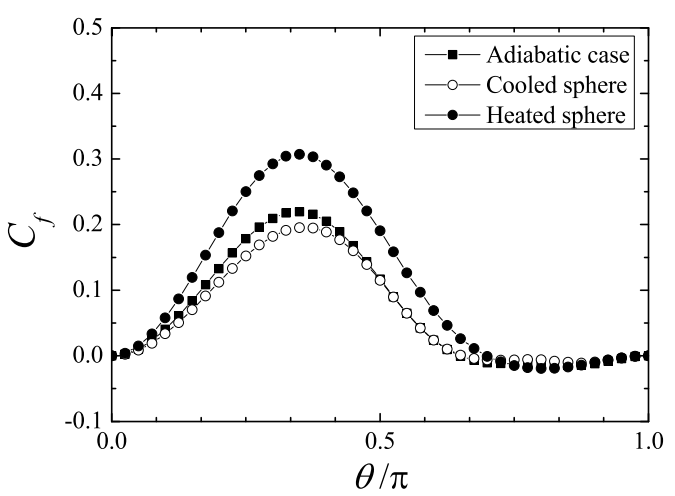

(a) Friction stress $C_{f}$

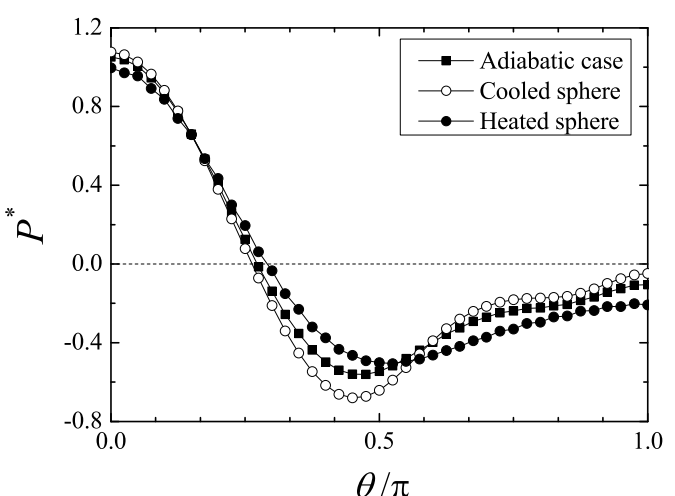

(b) Pressure $P^{*}$

Figure 9. Local friction stress $C_{f}$ and pressure $P^{*}$ on sphere surface for $R e_{p}=200$.

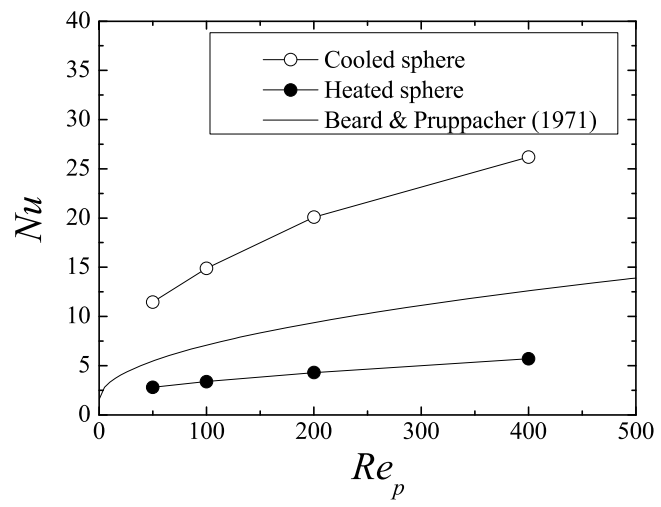

Figure 10. Nusselt numbers $N u$ against particle Reynolds number $R e_{p}$.

of the sphere:

$$
N u=\frac{d}{\left(T_{\text {sphere }}-T_{0}\right) S_{0}} \int_{S}\left(-\left.\frac{\partial T}{\partial r}\right|_{r=d}\right) d S,
$$

where $r$ is the normal component in spherical coordinates and $S_{0}$ is the surface area of the sphere. It is found that $N u$ of the heated and cooled spheres are smaller and larger than the empirical correlation (1971), respectively. This is because that the heated fluid in the vicinity of the sphere has the higher thermal conductivity $\lambda$, which enhances the thermal diffusion in fluid and therfore decreases the temperature gradient in the vicinity of the sphere surface. The exactly opposite phenomenon occurs for the cooled sphere.

\subsection{Presence of buoyancy}

\subsubsection{Vortex behavior}

Figure 11 shows the distributions of instantaneous streamlines and temperature on the $(x, y)$-plane for $R e_{p}=100$ and $d=50 \mathrm{~mm}$ in the presence of buoyancy. It is observed that for the heated sphere of $R i=0.5$ the vortex ring totally vanishes and tempera- 


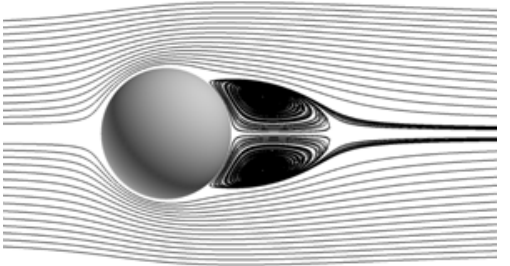

(a) Adiabatic case $(R i=0)$

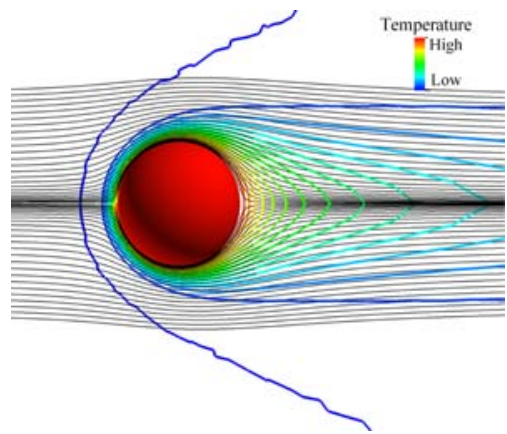

(b) Heated sphere $(R i=0.5)$

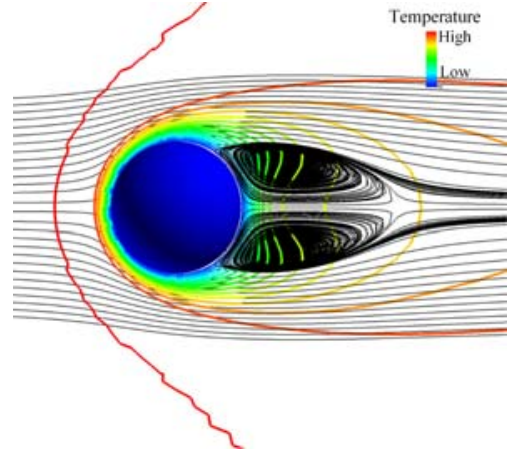

(c) Cooled sphere $(R i=-0.1)$

Figure 11. Distributions of instantaneous streamlines and temperaure for $R e_{p}=100$ and $d=$ $50 \mathrm{~mm}$ in the presence of buoyancy.

ture boundary layer around the sphere is extended to the downstream. This is because buoyancy acts to drive the heated fluid in the vicinity of the sphere surface to the downstream and hence the separation point of the heated sphere moves to the downstream. For the cooled sphere of $R i=-0.1$, on the other hand, the vortex ring is larger than that of the adiabatic case and the temperature boundary layer is contracted. This is because buoyancy acts to drive the cooled fluid in the vicinity of the sphere surface to the upstream and hence the separation point of the cooled sphere moves to the upstream. These tendencies of the heated and cooled spheres agree with the previous computations by Mansoorzadeh et al. (1998).

\subsubsection{Drag coefficient}

Figure 12 shows the drag coefficients $C_{D}$ of the heated and cooled spheres against Richardson number $R i$ for $R e_{p}=100$ for various sphere diameters of $1,5,10$ and 50 $\mathrm{mm}$ together with those of the adiabatic case and those obtained by the calculation with the Boussinesq approximation. For the calculation with the variable fluid properties, as absolute $R i$ increases, $C_{D}$ of the heated and cooled spheres increase and decrease from the value of the adiabatic case, respectively. Moreover, at a fixed $R i$, the deviations become notable as $d$ decreases with increasing $\Delta T$. These tendencies are mainly caused by buoyancy (i.e. the driving force on the fluid in the vicinity of the sphere surface) and the temperature dependencies of fluid properties (i.e. the change in viscosity which increases with increasing temperature), respectively. It is also found that only when the temperature difference is small enough in the case of large $d, C_{D}$ of both the heated and cooled spheres are well captured by the calculation with the Boussinesq approximation, since the Boussinesq approximation can consider only the effect of buoyancy without the temperature dependencies of fluid properties. 


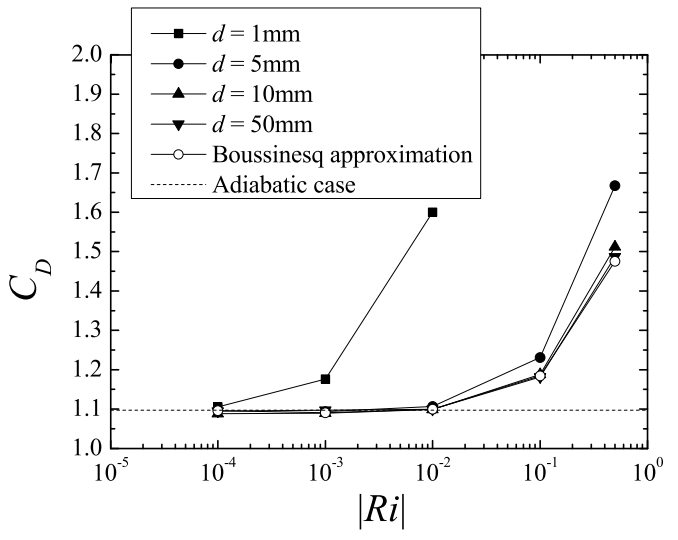

(a) Heated sphere

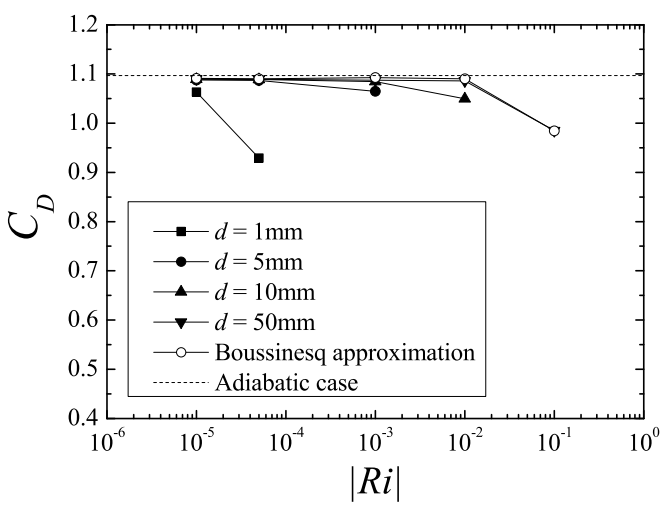

(b) Cooled sphere

FiguRE 12. Drag coefficients $C_{D}$ against Richardson number $R i$ for $R e_{p}=100$ in the presence of buoyancy.

\subsubsection{Nusselt number}

Figure 13 shows the Nusselt numbers $N u$ of the heated and cooled spheres against Richardson number $R i$ for $R e_{p}=100$ for various sphere diameters of $1,5,10$ and 50 $\mathrm{mm}$ together with those obtained by the calculation with the Boussinesq approximation and those obtained by the empirical correlation of Beard \& Pruppacher (1971). For the calculation with the variable fluid properties, as absolute $R i$ increases, $N u$ of the heated and cooled spheres increase and decrease, respectively, from the value of the empirical expression (1971) for the case of extremely small $\Delta T$ of $d=50 \mathrm{~mm}$, whereas they contrarily decrease and increase for the other cases. Moreover, at a fixed $R i$, the deviations become notable as $d$ decreases with increasing $\Delta T$. These tendencies are mainly caused by buoyancy (i.e. the expansion and contraction of the temperature boundary layer) and the temperature dependencies of fluid properties (i.e. the change in thermal conductivity $\lambda$ which increases with temperature), respectively. It is also found that as absolute $R i$ increases, $N u$ of the heated and cooled spheres obtained by the calculation with the Boussinesq approximation increase and decrease, respectively. These tendencies agree with those by the calculation with the variable fluid properties for the case of extremely small $\Delta T$ of $d=50 \mathrm{~mm}$, but not for the other cases. This is due to the fact that unlike the effects on $C_{D}$, the effects of buoyancy and the temperature dependencies of fluid properties on $N u$ act to change the values in the opposite way and the latter effect, which cannot be considered in the Boussinesq approximation, is dominant except for the conditions for the extremely small temperature differences.

\section{Conclusions}

The characteristics of flow past a heated/cooled sphere are investigated for $50 \leqslant R e_{p} \leqslant$ 400 in both conditions with and without buoyancy by measn of three-dimensional direct numerical simulation (DNS) in which temperature dependencies of fluid properties such as density and viscosity are exactly taken into account. The main results obtained in this study can be summarized as follows.

(1) In the absence of buoyancy, drag coefficients of the heated and cooled spheres are larger and smaller than those of the adiabatic case, respectively, and Nusselt numbers of them are smaller and larger than the values estimated by a widely used empirical 


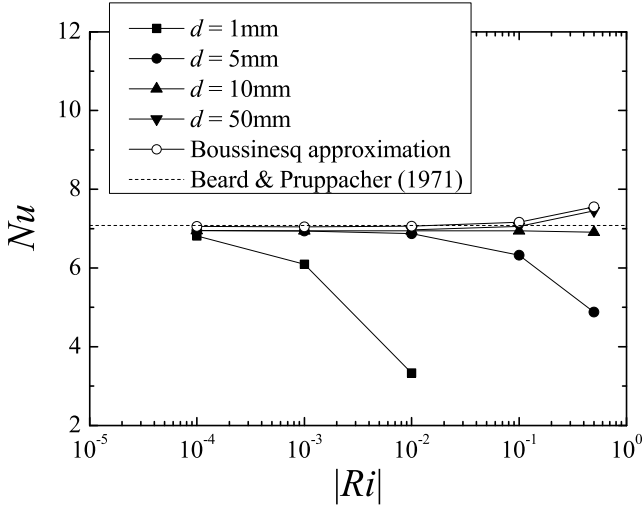

(a) Heated sphere

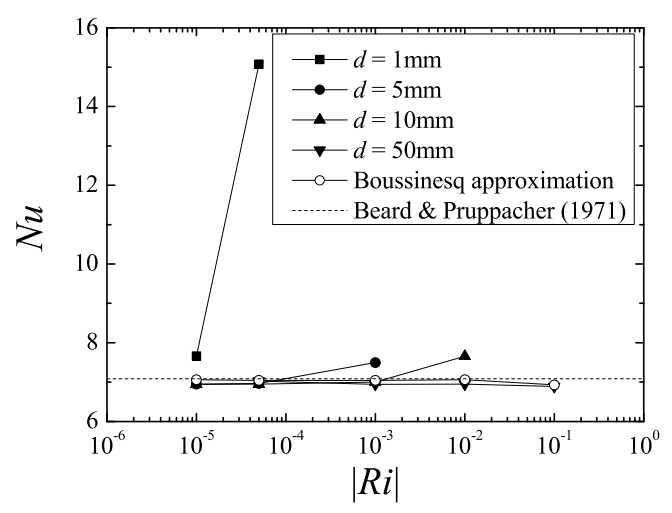

(b) Cooled sphere

Figure 13. Nusselt numbers $N u$ against Richardson number $R i$ for $R e_{p}=100$.

expression, respectively. In addition, the temperature difference between the sphere and ambient fluid strongly affects the flow separation points, size of vortex ring behind the sphere and Strouhal number for vortex shedding. These effects are attributed to the temperature dependencies of fluid properties in the vicinity of the sphere.

(2) In the presence of buoyancy induced by the gravity in the opposite direction of the flow, drag coefficients of the heated and cooled spheres increase and decrease from the values of the adiabatic case with increasing the absolute Richardson number, respectively. On the other hand, as the absolute Richardson number increases, Nusselt numbers of the heated and cooled spheres increase and decrease, respectively, from the values by the empirical expression for extremely small temperature differences between the sphere and ambient fluid, whereas they contrarily decrease and increase, respectively, for large temperature differences. Moreover, at a fixed Richardson number, the deviations in the drag coefficient and Nusselt number become notable with increasing the temperature difference. These tendencies are caused by the interaction between buoyancy and the temperature dependencies of fluid properties.

(3) The drag coefficients and Nusselt numbers of the heated and cooled spheres are predictable by the Boussinesq approximation only for small temperature differences, regardless of the magnitude of Richardson number.

The authors would like to thank Drs. Huilai Zhang and Kotaro Hori of Numerical Flow Designing, Ltd. for many useful discussions. This work was supported by Grant-in-Aid for Scientific Research (No. 20686015 and No. 22246020). This simulation was partially performed by super computer of National Institute for Environmental Studies, Center for Global Environmental Research.

\section{REFERENCES}

BabA, Y. \& Kurose, R. 2008 Analysis snd flamelet modelling for spray combution. J. Fluid Mech. 612, 45-79.

Bagchi, P. \& KotтAм, K. 2008 Effect of freestream isotropic turbulence on heat transfer from a sphere. Phys. Fluids 20, 073305.

Beard, K. V. \& Pruppacher, H. R. 1971 A wind tunnel investigation of the rate of evaporation of small water drops falling at terminal velocity in air. J. Atmos. Sci. 28, 1455-1464. 
Kee, R. J., Rupley, F. M. \& Miller, J. A. 1994 The chemkin thermodynamic data base. Sandia Report SAND87, 8215B.

Kotouč, M., Bouchet, G. \& DušEkc, J. 2009 A wind tunnel investigation of the rate of evaporation of small water drops falling at terminal velocity in air. Phys. Fluids 21, 054104.

Kurose, R., Fujita, A. \& Komori, S. 2009 Effect of relative humidity on heat transfer across the surface of an evaporating water droplet in air flow. J. Fluid Mech. 624, 57-67.

Kurose, R. \& Komori, S. 1999 Drag and lift forces on a rotating sphere in a linear shear flow. J. Fluid Mech. 384, 183-206.

Kurose, R., Makino, H. Komori, S. Nakamura, M. Akamatsu, F. \& Katsuki, M. 2003 Effects of outflow from surface of sphere on drag, shear lift, and scalar diffusion. Phys. Fluids 15, 2338-2351.

Mansoorzadeh, S., Pain, C. C., De Oliveira C. R. E. \& Goddard, A. J. H. 1998 Finite element simulations of incompressible flow past a heated/cooled sphere. Int. J. Numer. Math. 28, 903-915.

Nakamura, M., Akamatsu, F., Kurose, R. \& Katsuki, M. 2005 Combustion mechanism of liquid fuel spray in gaseous flame. Phys. Fluids 17, 123301.

SChiller, L. \& NAUman, A. 1933 Über die grundledede berechnungen bei der schwerkraftaufbereitung. Zeitschrift des Vereines Ceutscher Ingenieure 338, 325-357.

Sutherland, W. 1893 The viscosity of gases and molecular force. Philosophical magazine S.5, 36, 507-531.

TANEDA, S. 1956 Experimental investigation of the wake behind a sphere at low reynolds numbers. J. Physical Society of Japan 11, 1104-1108. 